\title{
Filigrane
}

Écoutes psychanalytiques

\section{Recension du livre L'amour de la différence, de Catherine Chabert}

\section{Lise Marceau}

Volume 25, numéro 1, printemps 2016

URI : https://id.erudit.org/iderudit/1037381ar

DOI : https://doi.org/10.7202/1037381ar

Aller au sommaire du numéro

Éditeur(s)

Revue Santé mentale au Québec

ISSN

1192-1412 (imprimé)

1911-4656 (numérique)

Découvrir la revue

Citer ce compte rendu

Marceau, L. (2016). Compte rendu de [Recension du livre L'amour de la différence, de Catherine Chabert]. Filigrane, 25(1), 169-177.

https://doi.org/10.7202/1037381ar d'utilisation que vous pouvez consulter en ligne.

https://apropos.erudit.org/fr/usagers/politique-dutilisation/ 


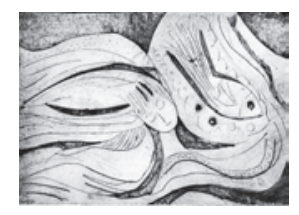

\title{
Recension du livre L'amour de la différence, de Catherine Chabert ${ }^{1}$
}

\section{Lise Marceau}

[...] entre le vif et le mort, la force d'attraction permet l'éclosion renouvelée de la recherche du plaisir. Oui, l'expérience prend ses sources dans l'ombre, oui, la méthode et la théorie affronte l'obscurité des profondeurs, mais nous le savons tous, il fait plus clair, il fait moins noir quand quelqu'un parle. (Chabert, 2011, p. 211)

C'est le plaisir qui préside au fonctionnement des processus inconscients. (Freud, 1920, p. 216)

\begin{abstract}
A u Congrès des psychanalystes de langue française à Montréal, en 2014, Catherine Chabert, citant Pontalis, nous disait que si la capacité de l'analyste à éprouver la souffrance était essentielle, son aptitude au plaisir ne l'était pas moins. Son livre L'amour de la différence (2011), qui collige des textes rédigés de 1992 à 2008, se termine par un chapitre intitulé «Le plaisir ». Plaisir qu'il ne faut pas confondre avec «les déclarations officielles du bonheur» (p. 227) ni avec «La cruelle contrainte au bonheur», titre de son avantdernier chapitre qui traite de la question du surmoi dans les deux sexes.

Un plaisir, par ailleurs, qui transpire de son écriture par la qualité littéraire de son style et les chassés-croisés multiples qu'elle fonde sur la référence à divers romans, personnages mythiques, fantasmes (tirés de textes de Freud et de Green), etc. Ce fut pour moi une lecture captivante d'un texte riche et dense, précédé d'un titre accrocheur - considérant la haine de toutes les différences qui sévit partout dans le monde. J'aborderai ici les différents thèmes qui me sont apparus fondamentaux, en commençant par un retour sur son ouvrage précédent.
\end{abstract}

\section{L'oubli du père}

Dans son livre précédent, Féminin mélancolique, publié en 2003, Catherine Chabert nous avait entretenus des fantasmes de l'enfant battu, 
de l'enfant mort, de mouvements mélancoliques, masochistes, de réaction thérapeutique négative et de patients qui ne veulent pas guérir. On aurait pu comprendre qu'elle éprouve, comme plusieurs, quelque pessimisme par rapport à l'avenir de la psychanalyse, mais ce n'est pas le cas. L'on trouve chez elle, en fait, une grande confiance en la méthode psychanalytique. C. Chabert avance avec Freud et à partir de Freud pour élaborer sa propre pensée, toujours solidement ancrée dans la clinique.

Du reste, bien qu'il ne s'agisse pas de pessimisme, l'on décèle tout de même une inquiétude par rapport à la psychanalyse contemporaine qui donne, selon elle, trop de place à la relation mère-enfant et oublie le père et avec lui, l'impact de la différence sexuelle. Oubli qui nous pousse, selon elle, du côté de la fusion et de «l'inceste (non séparé par définition)» et peut créer « un infléchissement de la méthode ainsi que de la théorie» (p. 56) qui risque de nous entraîner et de nous enfermer dans un mouvement de dédifférenciation. Un «rebroussement narcissique», dit-elle, avec sa «logique du mélange». Elle cite Jean-Luc Donnet, qui parle «d'une forme d'impersonnalisation du père et du surmoi dans la société occidentale contemporaine, susceptible de se retrouver dans le transfert». Cela risque «d'entraîner une désexualisation du fonctionnement psychique, [...] une abolition de la différence sexuelle» (p. 120-121).

D’ailleurs, le Féminin mélancolique se termine par un chapitre intitulé La femme qui avance, à l'instar de Gravida/Zoé analysée dans l'œuvre de Freud. «La femme qui marche, écrit-elle, la femme qui avance, [abandonne] les traces de son attachement mélancolique à sa mère morte, pour laisser son cours au plaisir de vivre.» (p. 181) Pour cela, il lui aura fallu passer de la mère au père libidinal qui serait « une seconde chance» dans sa double fonction de présence séductrice et de refus, respectueux de l'interdit de l'inceste, qui permettra les déplacements à venir vers la possibilité d'un nouveau partenaire amoureux engageant par là le déclin de l'œdipe (p. 180-181).

\section{L'amour de la différence}

Dans L'amour de la différence, C. Chabert reprend ce thème et nous fait part de son cheminement depuis 2003, qui l'a conduite à passer de l'angoisse de perdre l'amour de la part de l'objet dans sa version narcissique, «à une centration sur la chose sexuelle, c'est-à-dire, sur la différence des sexe» (p. 9). «Comme si, écrit-elle, hors de la grande dramaturgie odipienne, il n'y avait pas eu de lieu possible pour rassembler père et mère.» (p. 27) Dans la bisexualité psychique, telle que conçue par Freud, elle le répète, il n’y a 
pas de clivage entre féminin et masculin mais seulement «un peu plus de l'un que de l'autre», «une position d'emblée dialectique» (p. 30). Elle salue au passage Melanie Klein dont l'œuvre constitue, selon elle, «une référence majeure pour tenir ensemble, au sein des réseaux de la psycho-sexualité, les problématiques de la perte, la sexualité et la mort» (p. 191).

À l'instar de Freud qui définissait la psyché comme le Royaume de l'intermédiaire ou l'entre-deux (p.29), ce passage entre mère et père, elle l'appellera l'entre-eux-deux, qui représente non seulement un mouvement mais aussi «une ouverture» voire un espace (p. 31). Si le père libidinal offre une seconde chance d'investissement, un espoir, pour pallier une déception première et permettre de se dégager de l'emprise maternelle, il reste qu'il interdit l'inceste, qu'il sépare; il est l'empêcheur de tourner en rond. «Une autre forme, écrit-elle, de l'expérience transitionnelle» (p.31) puisque le père, objet sexuel de la mère, à la fois fait naître le désir mais aussi, l'interdit et la douleur de l'exclusion. La question paradoxale ne serait plus: cet objet l'ai-je inventé/créé ou était-il là?, mais deviendrait: est-ce mon désir ou le sien? Qui séduit qui?

C'est dans cet entre-eux-deux que C. Chabert situe le temps ou l'expérience de l'attente, qui est «ouverture associative et rêvante » à l'autre (p. 31). Temps «qui s'étire entre présence et absence», temps de la perte et du deuil «qui peut se confondre avec la solitude jusqu'à la détresse» (p. 23).

\section{De quelques cas cliniques...}

C'est ce que viendront illustrer avec brio les histoires cliniques que C. Chabert nous offre avec générosité ainsi que l'analyse de personnages empruntés à la littérature ou à la mythologie. Elle arrive à nous faire partager le mouvement même de la cure en même temps que celui de sa pensée. «L'histoire d'une analyse, écrit-elle, se crée à deux, patient et analyste et constitue, le noyau de l'intime, qu'il [...] est si difficile de transmettre sans être immédiatement saisi par la crainte d'une trahison». Elle adoptera alors «la voie de la fiction qui éloigne de l'idée de traitrise et permet d'approcher non pas la vérité mais l'expérience, au sens de ce qui s'éprouve» (p. 16).

J'ai été intriguée tout au long de ma lecture par ces histoires cliniques où elle parle de: «moment crucial», «moment hallucinatoire» qui déterminent des «tournants» ou des dénouements dans le processus analytique en cours, qui fait que l'analyste effacée dans son altérité et sa différence sexuelle réapparaît, ou que «le père advient», ou que «son amour est retrouvé». Des moments où quelque chose surgit qui participe à la fois du familier, de 
l'intime et de l'étranger (Unheimliche)... Comme si la magie y était sollicitée mais pour laisser place à une autre magie: celle de la symbolisation qui fait se différencier soi et monde, d'où l'apparition d'une nouvelle perspective à partir de laquelle une histoire peut se construire. Des moments, source de plaisir puisque le mouvement de la vie et de la pensée y est promu. C'est ce que j'ai tenté d'explorer à travers les cures de Violette et d'un cas de jalousie qui sont particulièrement élaborés.

\section{Violette}

Violette était venue consulter parce qu'ayant perdu un de ses deux amants, elle avait le sentiment d'avoir tout perdu. Elle avait perdu les mots et ne lui restait que l'angoisse. Elle choisit une cure de paroles. Après la remémoration/ répétition incessante de la scène des coups au visage administrés par le père, répétition qui avait pour effet de paralyser l'analyste ( Je me sentais impuissante et écrasée par les faits», p. 63), la scène se déplacera enfin dans le cadre de porte de la chambre d'enfant de Violette. Dès lors, l'analyste sera transportée dans cette chambre telle qu'elle l'imagine. "Je sus assez vite, écrit-elle, que cette chambre ressemblait à celle de mon enfance. Voilà que Violette et moi partagions un espace, en pensée.» (p. 63) Dans la chambre des souvenirs d'enfance de l'analyste, loin du lieu d'une scène traumatique où Violette l'emmenait, l'analyste écrit: "La nostalgie, la douceur de la remémoration s'immisçait subrepticement. Cela, indique-t-elle, marqua un tournant dans la cure.» (p. 67) En effet, à ce moment-là, Violette rêve qu'elle brise un appareil photo. Finis les arrêts sur images et «l'emprise des faits» (p. 73); «J'eus le sentiment, écrit C. Chabert, de sortir d'un tunnel.» (p. 64) L'énergie circula à nouveau.

Il fallut donc passer par L'enterrement du compte d'Orgaz, un tableau de Greco, Peau d'âne, la tragédie des Atrides, le drame d'Iphégénie, pour permettre à Violette de penser la violence des scènes et retrouver l'amour de et pour son père, de même que la capacité d'affronter sa mère oedipienne. Ce faisant, elle put tisser les fils de son histoire et en être l'auteure.

«L'analyste, écrit C. Chabert, rêve les rêves de ses patients par la voie de sa propre associativité.» (p. 64) Du mot à l'image jusqu'à la sensation. Dans ce chapitre, d'ailleurs, C. Chabert illustre de façon dynamique à quel point la scène, comme le souvenir, vient faire écran au fantasme, mais aussi à «sa matière première, la sensation qui, en tant que perception interne de ce qui s'éprouve dans le plaisir et le déplaisir, en constitue la source» (p. 67). «En fait, précise $\mathrm{C}$. Chabert, nos souvenirs conscients ne sont pas des souvenirs d'enfance, ce sont des souvenirs sur notre enfance.» (p. 67) 


\section{Monsieur IL}

Dans le chapitre intitulé «Logique inconsciente de la différence», l'auteure rapporte un cas de jalousie, mais ne nomme pas son patient. Elle écrit: «Il est venu me consulter». Je l'appellerai donc M. IL.

M. IL vient la consulter pour une jalousie maladive qui est apparue récemment à l'occasion d'une liaison passionnelle avec une femme. Sa sœur aînée puis sa mère étaient décédées peu avant. M. IL ne les aimait pas. Sa mère préférait les filles.

De son côté, l'analyste avait commencé la lecture d'un roman de Mendelsohn intitulé Les disparus. Chemin faisant, avec M. IL, elle se sentit disparaître, effacée dans le transfert en tant que femme sexuelle, écrit-elle. Dans son roman, Mendelsohn qui détestait son frère cadet dont il était jaloux, cherche la trace de la haine fraternelle et de ses excès. Il montrera que sous la haine couve l'amour, et une relecture de Freud - De quelques mécanismes névrotiques dans la jalousie, la paranoïa et l'homosexualité (1921) - lui rappellera que les anciens rivaux deviennent des objets d'amour homosexuels.

Pendant ce temps, M. IL rêve d'une femme dont la moitié du visage est roussie et l'autre intacte. L'analyste pense au film L'Allemagne, mère blafarde, mais elle ne dit rien. Puis M. IL rêve à des généraux nazis et à sa soumission qui perdure avec son père. À son tour, il évoque ce même film, et en particulier la scène où une petite fille crie pour empêcher sa mère de se tuer; scène que de son côté, l'analyste avait oubliée.

À ce moment, M. IL fond en larmes et sa jalousie se déchaîne, mais «il protège [l'analyste] absolument de ses attaques ». Plus que jamais, C. Chabert se sent disparue, effacée en tant que femme sexuelle, mais aussi en tant que sujet: «[...] j'ai le sentiment de ne pas exister vraiment, une momie ou un robot toujours-là, un transfert invisible.» (p. 114)

C'est alors que M. IL réalise ou prend conscience de sa jalousie meurtrière envers sa sœur décédée. Il rêve qu'il fait l'amour avec une femme homosexuelle. Il a un vagin tout en conservant son pénis. Il prend conscience de l'amour qu'il porte à son analyste. "Vous êtes ma première femme... ", avaitil échappé en s'étendant sur le divan au tout début. À la suite de cet aveu, ou de ce retour du dénié-clivé, l'analyste put penser l'effacement qu'elle avait éprouvé comme «meurtre de la part étrangère de l'objet» (p. 117), «comme détournement libidinal» et «agrippement à un double pour lutter à tout prix contre la différence et la jalousie qu'elle génère» (p. 116).

Cette fois-ci, C. Chabert questionne cette association commune; ce phénomène de transmission. L'Allemagne, terre blafarde, transmission de pensée? 
se demande-t-elle. Phénomène «télépathique» qui annule les frontières entre l'analyste et l'analysant, créant une brèche où se faufile du même? Peut-être, répond-elle, mais nos chemins se dispersent et divergent. Elle ajoute que cette expérience transférentielle relève de «cette forme hallucinatoire si particulière que représente l'association à une œuvre artistique» (p. 118).

\section{L'homosexualité primaire en double}

J'aimerais partager une réflexion au sujet de ces phénomènes télépathiques que je tenterai de formuler à partir de la lecture du texte de Roussillon: La dépendance primitive et l'homosexualité primaire en double (2004), formule qu'il reprit d'Evelyne Kestemberg, citée par C. Chabert dans le chapitre intitulé "Ce que mon père préfère...» Dans ce chapitre, C. Chabert déconstruit le mythe de Laïos et le fantasme de pédophilie pour en faire «le noyau du fantasme de séduction» (p. 168) ou encore «l'énigme au commencement de l'amour» (p. 161). L'histoire de Laïos et à sa suite, celle d'CEdipe-roi, répètent le même scénario de transgression, d'annulation de la différence des sexes et des générations et de mise à nu de l'inceste. Cependant, «une des composantes du mythe retiendrait l'idée de la pédophilie - l'amour de l'enfance - comme investissement narcissique primordial, fondement essentiel à toute construction identitaire et à tout mise en mouvement de la relation d'objet» (p. 165), «[...] à l'origine des origines, comme référence primordiale de l'organisation de drame œedipien» (p. 161).

C'est avec le concept de relation primitive homosexuelle en double que Roussillon, à la suite de Kestemberg, tente de caractériser le narcissisme primaire et l'expérience de satisfaction entre la mère et l'infans. Cette «relation primitive», «ballet» ou «chorégraphie» de la rencontre, selon Roussillon, «suppose que l'autre primordial soit rencontré d'abord comme un semblable $[. .$.$] qu'il accepte [. .$.$] de réfléchir» et de "partager les mêmes états$ d'être, les mêmes états d'âmes [...] Il se propose donc comme un double, un autre semblable mais tout en restant soi» (Roussillon 2004, p. 423). Dans cette expérience de partage de plaisir, que Kestemberg qualifie «de véritables échanges amoureux» (Chabert, 2011, p. 165), l'un et l'autre des deux partenaires se constituent comme miroir. Cela favorisera l'accordage esthésique et affectif que l'on sait mais à la condition incontournable qu'il n'y ait pas de confusion entre l'un et l'autre. En effet, le plaisir sexuel infantile et le plaisir génital adulte ne sont pas la même chose. Les satisfactions sexuelles que la mère peut éprouver la renvoient à ses imagos œedipiennes et sont transformées "plus ou moins consciemment rapidement en caresses tendres, 
“épurées” (Kestemberg, 1984, p. 9). Côté enfant, «le plaisir qu’il trouve en lui-même et celui qu'il trouve dans l'échange ont une qualité différente» (Kestemberg, 1984, p. 15). Cette différence de qualité, précise Kestemberg (1984), est "vécue sensoriellement» et constitue «la base de la satisfaction hallucinatoire et de l'insatisfaction qui lui est inhérente» (p. 15); elle rend possible la construction de représentants psychiques (Roussillon, 2004). Ainsi, ce qui différencie fait lien puisqu'est activée la fonction de symbolisation. On pourrait dire que sur la scène du réel, le père est garant de cette différence et de la fonction symbolique. Finalement, c'est tout ce bal qui déterminera le jeu des identifications et des investissements d'objet. «[...] tout le travail psychique de l'homosexualité, écrit Kestemberg, est d'organiser l'altérité pour à travers elle conserver l'identité» (Chabert, 2011, p. 165-166).

Encore faut-il que cette différence dans la qualité du plaisir sexuel, que le manque ou le sentiment de perte qu'elle implique, soit supportable. «C'est parce que l'autre est d'abord conçu comme un "double", écrit Roussillon, que la différence peut être construite autrement que comme une forme de clivage, que comme une forme de répudiation» (2011, p. 190), comme on a pu le voir dans le cas de M. Il.

La censure de l'amante (Braunschweig, 1975) qui porte la jouissance du couple et les imagos œdipiennes introduit donc, dans cette «chorégraphie» de la rencontre, une différence énigmatique qui n'aura jamais fini de livrer son sens ou de trouver une traduction. "L'énigme est originaire», écrit C. Chabert (2011, p. 160). Le vécu sensoriel qu'elle détermine, la sensation, est la matière même du fantasme et du transfert. «[...] le mythe (collectif et verbal) comme le fantasme (individuel), écrit-elle, vient dramatiser, mettre en scène la réponse à une énigme, donner une figuration, et peut-être un sens, au trouble, à l'émoi aux bouleversements induits par l'émergence de la question» (p. 167).

Ainsi, la différence sexuelle, comme représentante de la séparation et en même temps, comme lien entre l'un et l'autre, est au fondement de nos psychismes. Nous comprenons mieux la pertinence du livre de Catherine Chabert et la portée de son titre: L'amour de la différence.

\section{Retour à la clinique}

Dans le cas de Violette et celui de Monsieur IL, analyste et analysant se retrouvent dans un même lieu psychique, chambre d'enfant ou image de film. Comme s'il fallait retourner, régresser à ce niveau infra-linguistique de «la relation primaire homosexuelle en double», pour que «la parole du 
patient résonne dans le cœur des représentations de l'analyste», afin que surgisse l'interprétation ou le mot juste qui viendra rompre les charmes ou le magnétisme de la captation imaginaire à l'objet primaire, qui permettra le dégagement de la répétition de l'identique et l'apparition d'une nouvelle vision du monde. C'est aussi ce qui déterminerait ces fameux tournants ou virages dans la cure, qualifiés parfois de télépathie, que j'aurai tenté d'élucider. Moment fugitif et éprouvant, le surgissement du «mot juste» au cœur de l'écriture comme de l'interprétation, signe «la rencontre de ce qui se dit avec ce qui s'éprouve» (p. 22). «[...] c'est dans ces moments d'extrême solitude, écrit C. Chabert, quand l'absence et la perte, quand la détresse ou la cruauté envahissent la situation analytique, qu'une sorte de réalisation hallucinatoire du fantasme s'empare de l'analyste, [...]: l'image et le mot viennent alors prêter une forme à un état d'affect bouleversant qui brouille les repères» (p. 209). «Au lieu d'une place vacante créée par l'absence ou la perte, la représentation émerge, et son pouvoir attractif, séducteur, transporte et ranime la trace libidinale laissée par l'objet perdu.» (p. 224) Si, par ailleurs, l'objet ou l'analyste ne reflétait qu'un silence opaque ou une fin de non-recevoir, une partie de soi serait séquestrée dans l'autre, observe Roussillon, d'où une zone de non-différenciation de l'objet et du sujet donnant lieu à une identification adhésive (Esther Bick), ce qui rendrait impossible le deuil de l'objet, puisque renoncer à l'objet serait alors renoncer à la part de soi séquestrée en lui (Roussillon, 2011, p. 189).

\section{Conclusion}

Plaisir de l'écriture, plaisir de la lecture. Masculin? Féminin? Le livre illustre sur la scène de l'écrit le lien qui unit féminin et masculin. En effet, ce livre porte un argumentaire masculin, ne serait-ce que par sa référence soutenue à Freud et par le cadre même du livre, la langue, la règle, la séparation... Il porte en même temps à travers l'écriture, le style et les histoires cliniques — qui se lisent comme des romans —, la part du féminin créatif de l'auteur.

Ce féminin-là, qu'elle nomme et qu'on retrouve aussi bien chezl'homme. «Dans son creux, écrit-elle, le féminin abrite et condense les représentations de l'enfant, de l'enfance, de l'infantile sans doute par l'attraction d'images de passivité, d'impuissance et de désarmement ou encore par un infléchissement probable vers la pénétration ou l'intrusion. [...] ce féminin-là constitue le point de sédimentation essentiel de la bisexualité et des identifications qui en découlent.» (Chabert, 2003, p. 12) Entre la menace de l'identique et 
l'angoisse de castration, entre la perte et l'espoir, le féminin soutient l'expérience de l'attente inhérente à la position ou à l'écoute de l'analyste, le temps, pour ce dernier, de se laisse traverser, pénétrer par les scènes, mots, images de patient(e) jusqu'à la sensation, jusqu'à l'expérience hallucinatoire du fantasme laquelle, on le sait maintenant, initie des tournants ou des virages... pour autant que l'analyste tienne la route... L'analyste écoute? Non il attend.

C. Chabert termine son livre en beauté. En effet, le dernier chapitre s'intitule: Le plaisir. Le plaisir de séance notamment, entre les deux protagonistes, ce plaisir, qui «tisse la trame de nos désirs et de nos rêves, [...] celui qui fait venir pendant des mois, des jours, des années, dans le même lieu et aux mêmes heures, les deux invités dont la présence est requise pour que la séance commence» (p. 227).

«L'invention de Freud, rappelle-t-elle, c'est l'invention de la psychosexualité à travers l'étude du plaisir, de ses entraves, de son contraire et de son au-delà.» (p. 210)

Lise Marceau marceaul@videotron.ca

\section{Note}

1. Texte élaboré à partir d'une présentation par l'auteure du livre de Catherine Chabert, à l'occasion de la journée clinique organisée par la Société psychanalytique de Québec, en octobre 2015.

\section{Références}

Braunschhweig, D. et Fain, M. (1975). La nuit, le jour, Essai psychanalytique sur le fonctionnement mental. Paris: Presses universitaires de France.

Chabert, C. (2003). Féminin mélancolique. Paris: Presses universitaires de France.

Chabert, C. (2004). La voix du père: une seconde chance. Dans L'oubli du père (p. 11-20). Paris: Presses universitaires de France.

Chabert, C. (2011). L'amour de la différence. Paris: Presses universitaires de France.

Chabert, C. (2015). La jeune fille et le psychanalyste. Paris: Dunod.

Freud, S. (1920). Au-delà du principe de plaisir. Euvres complètes tome XV - 1916-1920 (p. 273-338). Paris: Presses Universitaires de France, 2002.

Kestemberg, E. (1984). «Astrid» ou homosexualité, identité, adolescence. Quelques propositions hypothétiques. Les Cahiers du centre de psychanalyse et de psychothérapie, (8), 1-30.

Roussillon, R. (2004). La dépendance primitive et l'homosexualité "primaire» en double. Revue française de psychanalyse, 68 (2), 421-439.

Roussillon, R. (2004a). Le reflet et son négatif. Libres cahiers pour la psychanalyse, 2 (10), 73-85.

Roussillon, R. (2011). Déconstruction du narcissisme primaire. L'année psychanalytique internationale, 1, 177-193. 